\title{
Hospital admissions from a pediatric HIV care and treatment program in Malawi
}

\author{
Carl A. Nosek ${ }^{1,2^{*}}$, W. Chris Buck ${ }^{1,3}$, Alison C. Caviness ${ }^{4}$, Abbie Foust ${ }^{5}$, Yewo Nyondo ${ }^{1}$, Madalitso Bottomani ${ }^{1}$ \\ and Peter N. Kazembe
}

\begin{abstract}
Background: The scale up of pediatric antiretroviral treatment programs across Sub-Saharan Africa over the last decade has brought increasing numbers of children into HIV care. This patient population requiring life-long care presents new challenges in the outpatient and inpatient settings. We sought to describe hospitalizations from a large pediatric HIV treatment facility to better understand the scope of the situation and identify areas for improved care delivery.

Methods: We conducted a retrospective case series of all HIV-infected and exposed patients $<18$ years enrolled at Baylor College of Medicine Children's Foundation Malawi, from October 2004-October 2010. Patients admitted to the hospital on or after the day of enrollment were included. Data were extracted from electronic clinic records. Analysis was done at the patient and admission level, as some patients had multiple admissions.

Results: Of 5062 patients enrolled in care, 877 (17.3 \%) had 1137 admissions at median age 24 months (IQR: 12-62). 191 (21.8\%) patients had multiple admissions. A high proportion of admissions occurred in patients under two years (49.4\%), those within one month of clinic enrollment (32.9\%), those with severe immune suppression (44.0\%), and those not on ART (48.5\%). The frequency of primary admission diagnoses varied across these same variables, with malnutrition, pneumonia, and malaria being the most common.

Conclusions: Illness requiring hospitalization is common in HIV-infected and exposed children and these results reinforce the need for a comprehensive care package with special attention to nutrition. Strengthened programs for malaria prevention and expanded access to pneumococcal vaccine are also needed. The high burden of admissions in children under 24 months and those newly enrolled in care suggests a need for continued improvement of early infant diagnosis and provider-initiated testing programs to link patients to care before they are symptomatic. Similarly, the high proportion of admissions in those not yet started on ART emphasizes the importance of rapid initiation of ART for eligible pediatric patients.
\end{abstract}

Keywords: HIV, Pediatric, Admission, Hospitalization

\section{Background}

Sub-Saharan Africa has the highest burden of pediatric HIV in the world, with over $90 \%$ of the estimated 3.2 million children living with HIV globally. The last decade has seen a massive scale-up of antiretroviral treatment (ART) programs in the region, and 551,065 children were reported to be receiving ART in subSaharan Africa at the end of 2012 [1]. Supporting these

\footnotetext{
* Correspondence: canosek@yahoo.com

'Baylor Children's Foundation Malawi, Lilongwe, Malawi

${ }^{2}$ Department of Pediatrics, University of California San Francisco, San

Francisco, California, USA

Full list of author information is available at the end of the article
}

huge numbers of patients on life-long ART is increasingly stressing already weak national health systems, and the challenges in the chronic outpatient management of HIV are well-documented [2, 3].

Much less has been published about the impact of the pediatric HIV epidemic on inpatient facilities that care for children. Studies done prior to the roll-out of national ART and prevention of mother to child transmission (PMTCT) programs reported very high HIV prevalence in admitted children with corresponding high inpatient mortality rates (29 and $17 \%$, respectively in one study from Soweto in 2000) [4-9]. More recent 
research has demonstrated decreasing, but still overall high HIV prevalence and associated mortality in pediatric admissions (19 and $12 \%$, respectively in a follow-up study from the same hospital in Soweto in 2010) $[10,11]$.

Despite this progress, regional pediatric ART coverage rates are still very low (only $32 \%$ of eligible infected children were on treatment in 2012), and much of the inpatient HIV burden likely still comes from children not yet enrolled in care and treatment [1]. However, as national ART programs continue to expand and mature, and vertical transmission rates continue to decrease, it is probable that a larger percentage of hospitalized HIVinfected children will already be enrolled in HIV care. There is very little published literature on this subject using the outpatient HIV care clinic as the starting point for analysis, rather than the pediatric ward [12].

Table 1 Characteristics of 877 patients admitted to the Hospital, Malawi 2004-2010

\begin{tabular}{|c|c|c|}
\hline & Number & Percent \\
\hline \multicolumn{3}{|l|}{ Sex } \\
\hline Female & 410 & 46.8 \\
\hline Male & 467 & 53.2 \\
\hline \multicolumn{3}{|c|}{ Number of Admissions } \\
\hline 1 & 685 & 78.1 \\
\hline 2 & 152 & 17.3 \\
\hline 3 & 21 & 2.4 \\
\hline $4-8$ & 19 & 2.2 \\
\hline \multicolumn{3}{|c|}{ Patient Status as of October 2010} \\
\hline Active & 393 & 44.8 \\
\hline Died & 270 & 30.8 \\
\hline Lost to follow-up & 95 & 10.8 \\
\hline Transferred out & 119 & 13.6 \\
\hline \multicolumn{3}{|c|}{ Age at First Admission } \\
\hline$\leq 11$ months & 218 & 24.9 \\
\hline $12-23$ months & 229 & 26.1 \\
\hline 24-35 months & 106 & 12.1 \\
\hline 36-59 months & 101 & 11.5 \\
\hline 60-119 months & 117 & 13.3 \\
\hline 120-179 months & 78 & 8.9 \\
\hline$>180$ months & 28 & 3.2 \\
\hline \multicolumn{3}{|c|}{ Time from Clinic Enrollment to First Admission } \\
\hline First Visit & 208 & 23.7 \\
\hline $1-30$ days & 157 & 17.9 \\
\hline $31-90$ days & 163 & 18.6 \\
\hline 90-365 days & 207 & 23.6 \\
\hline$>365$ days & 142 & 16.2 \\
\hline
\end{tabular}

In this context, we conducted a retrospective review of hospital admissions from a large cohort of children receiving HIV care in Malawi, seeking to characterize this specific patient population and better understand the implications for both the inpatient and outpatient settings.

\section{Methods}

This was a retrospective case series of all children $(<18$ years) who were enrolled in care at the Baylor College of Medicine-Abbott Fund Children's Clinical Centre of Excellence (COE) between October 2004 and October 2010 and had a documented hospital admission in their outpatient records. The COE provides comprehensive HIV care (including TB treatment, supplemental and therapeutic foods for malnutrition, and sick visits) to patients in the Lilongwe area, serves as a national HIV referral center, and is the largest provider of pediatric ART in Malawi with approximately $8 \%$ of all children on treatment at the time of this analysis [13]. The COE, an outpatient facility, is located immediately adjacent to Kamuzu Central Hospital $(\mathrm{KCH})$, the regional referral hospital, which has about 14,000 pediatric admissions per year and a previously reported pediatric inpatient HIV-infection and exposure prevalence of 8.5 and $6.5 \%$ respectively $[11,14]$. There is a strong referral system between the $\mathrm{COE}$ and $\mathrm{KCH}$, both for admissions from clinic as well as referrals for outpatient HIV care for those newly diagnosed on the wards. Clinicians from the $\mathrm{COE}$ also provide HIV consultative services on the $\mathrm{KCH}$ pediatric wards.

All study data came from the COE's outpatient electronic medical record (EMR). Paper-based inpatient $\mathrm{KCH}$ records were not used to supplement the information contained in the EMR, as it was not logistically feasible to link them to clinic files. Patients with a documented admission on or after the day of registration in HIV care at the COE were eligible for inclusion. All HIV-infected children were eligible for inclusion. Patients who were enrolled as exposed and later tested definitely HIV-infected were also included, as were infants who were still considered exposed without definitive HIV testing as of Oct. 2010. The only patients who were excluded based on HIV status were those who enrolled as exposed, but were later discharged as definitively HIV-uninfected. All patients enrolled in the COE have electronic medical records and no patients were excluded for lack of records.

A list of patients with documented admissions was generated by an EMR query. Patient EMR records for each admission (including post-hospital discharge records when available) were then manually reviewed to collect study data including primary admission diagnosis and other secondary admission diagnoses. Acute nutritional 
Table 2 Frequency of primary and overall diagnoses for 1137 admissions, Malawi 2004-2010

\begin{tabular}{lll}
\hline & Primary diagnosis N (\%) & All diagnoses N (\%) \\
\hline Malnutrition & $302(26.6 \%)$ & $401(13.6 \%)$ \\
Pneumonia & $212(18.6 \%)$ & $402(13.6 \%)$ \\
Malaria & $112(9.9 \%)$ & $233(7.9 \%)$ \\
Gastroenteritis & $68(6.0 \%)$ & $294(10.0 \%)$ \\
Sepsis & $68(6.0 \%)$ & $330(11.2 \%)$ \\
Other Infection & $53(4.7 \%)$ & $86(2.9 \%)$ \\
PCP Pneumonia & $48(4.2 \%)$ & $74(2.5 \%)$ \\
Tuberculosis & $45(4.0 \%)$ & $150(5.1 \%)$ \\
Kaposi Sarcoma & $43(3.8 \%)$ & $66(2.2 \%)$ \\
Anemia & $38(3.3 \%)$ & $174(5.9 \%)$ \\
Neurologic & $28(2.5 \%)$ & $78(2.6 \%)$ \\
Oncologic & $19(1.7 \%)$ & $21(0.7 \%)$ \\
Pulmonary (including LIP) & $16(1.4 \%)$ & $38(1.3 \%)$ \\
Medication Adverse Effect & $15(1.3 \%)$ & $18(0.6 \%)$ \\
Candidiasis & $8(0.7 \%)$ & $393(13.3 \%)$ \\
Hematologic & $5(0.4 \%)$ & $78(2.6 \%)$ \\
Cryptococcal Meningitis & $4(0.4 \%)$ & $5(0.2 \%)$ \\
Other & $36(3.2 \%)$ & $95(3.2 \%)$ \\
Unknown & $17(1.5 \%)$ & $17(0.6 \%)$ \\
TOTAL & $1137(100 \%)$ & $2951(100 \%)$ \\
\hline
\end{tabular}

status (per Malawi guidelines using weight/length, mean upper arm circumference (MUAC,) and edema assessments), TB status, WHO staging, and CD4 closest in time to admission (+/ 6 months from admission date) were also recorded [15]. These data were entered into an Access $^{\oplus}$ database (Microsoft Corporation, Redmond, WA, USA) and merged with other clinical and demographic data extractable from the EMR without chart review.

Data analysis was performed using SPSS 20.0 (IBM, Chicago, IL) and was primarily descriptive. Continuous variables were categorized for further description using frequencies. Patient age was categorized as 0-11, 12-23, $24-35,36-59,60-119,120-179$, greater than and equal to 180 months. Patient age was also described using medians and interquartile ranges. CD4 results were stratified according to WHO age-based immune classifications, with preferential use of CD4\% in children less than 5 years, and absolute CD4 in those greater than and equal to 5 years [16]. Time since enrollment was categorized into first visit, 1-30, 31-90, 90-365, and greater than 365 days. Time on ART was categorized into none, less than $1,1-2,3-6,7-12$, and greater than 12 months. All other patient and admission-level variables were categorical and described using frequencies.
Analysis was done primarily using admissions as the base variable since some patients had more than one admission and variables such as ART status, age, immune status, etc. varied from one admission to another. Also, since many admissions were associated with multiple diagnoses, the frequency of admission diagnoses was described separately as primary (as identified from the EMR) and overall (any diagnosis for the admission, independent of whether it was primary). Stacked bar graphs were used to graphically describe the frequency of primary admission diagnoses within categories of patient age, time from enrollment in HIV care, immune status, and time from ART initiation.

This study was conducted as part of a general retrospective EMR review protocol that was approved by both the Baylor College of Medicine and Institutional Review Board and the Malawi National Health Sciences Research Committee. Under these ethics approvals, informed consent was not required for retrospective analysis of de-identified routine clinical care data.

\section{Results}

A total of 877 individual patients (median age 24 months (IQR: 12-62), $46.8 \%$ female, $53.2 \%$ male) were identified as having 1137 separate admissions, representing approximately $17.3 \%$ (877/5062) of the comparable clinic population ever enrolled. The large majority of patients $(685 / 78.1 \%)$ only had one documented admission, while multiple hospitalizations were documented for 192 (21.9\%) patients, and 19 (2.2\%) had four or more admissions. Patients with an oncologic diagnosis on their first admission were more likely to be admitted more than one time (OR 6.1, $95 \%$ CI 1.4-25.7) over the duration of the study. No other first-admission variable (age, WHO stage, immune status, nutritional status, ART, TB, or diagnosis) was significantly associated with multiple admissions.

Among this group of admitted patients, 393 (44.8\%) were alive and in care at the end of the study period, 271 (30.9\%) had died (not necessarily during the hospital admission), 119 (13.6\%) had transferred out to other facilities/outpatient clinics, and 94 (10.8\%) were lost to follow up as of October 2010. Additional patientlevel data is found in Table 1.

Analysis of admission-level data revealed that almost half $(562,49.4 \%)$ of the total 1137 admissions occurred in patients under two years of age. Related to the time from clinic enrollment, 207 (18.2 \%) occurred on the child's first visit and 374 (32.9\%) occurred within one month. Of the 551 admissions in HIV-infected patients not yet on ART, 216 (39.2\%) were enrolled in the previous 14 days and 286 (51.9\%) in the previous 30 days. 


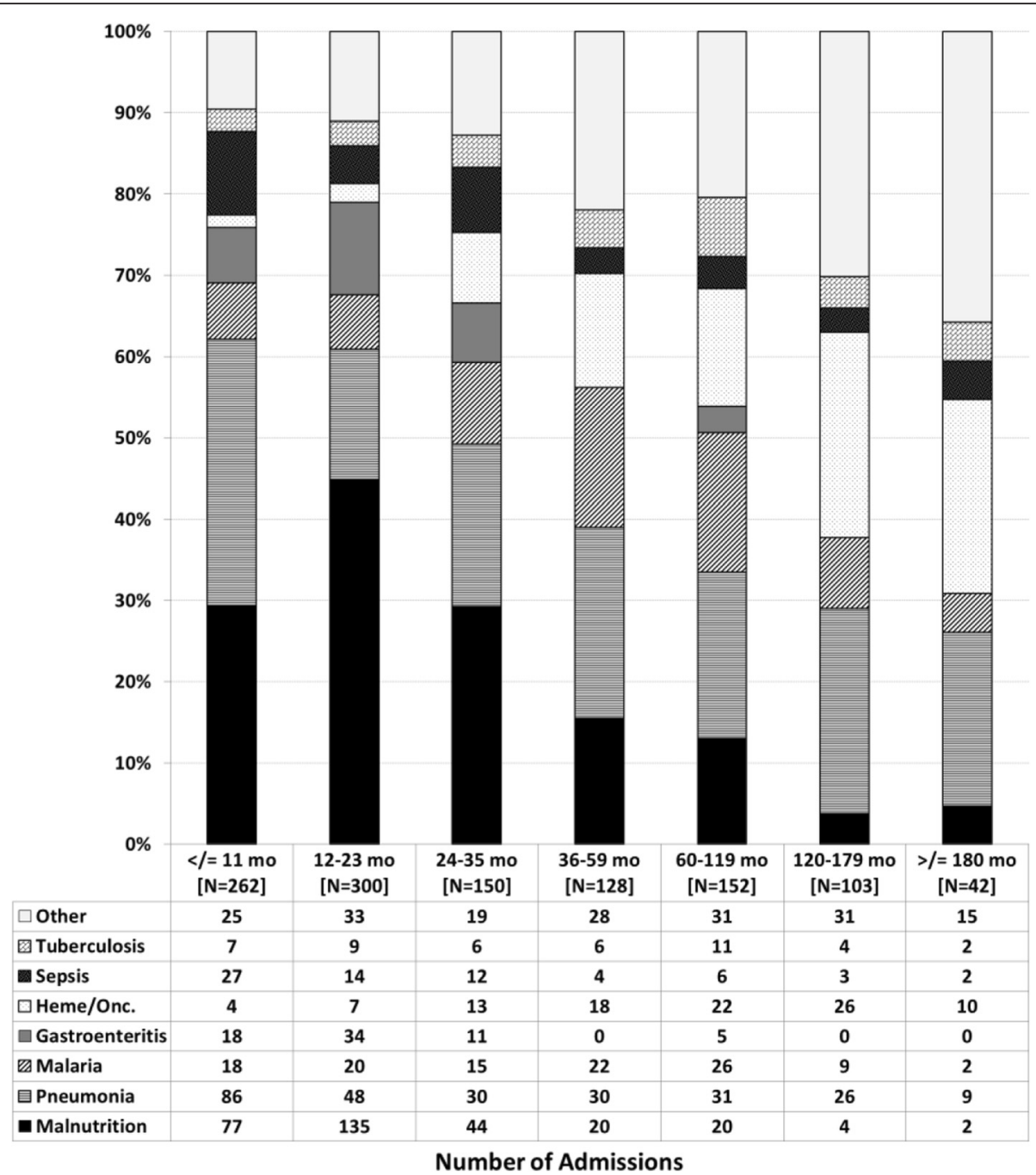

Fig. 1 Stacked bar graph of primary admission diagnosis by age group, Malawi 2004-2010

Most admissions were in patients with advanced clinical staging (934, $82.1 \%$ were WHO III or IV.) Severe suppression was the most common immune status at admission (501, 44.0\%), however 230 (20.2 \%) of admissions were in patients with no immune suppression.

Many admissions were associated with multiple diagnoses - there were 2951 total diagnoses identified for the 1137 admissions. Malnutrition and pneumonia were the most common primary diagnoses (26.6 and $18.6 \%$, respectively) and overall diagnoses (13.6 and $13.6 \%$ respectively (Table 2 ).

The relative frequency of primary admission diagnoses differed based on various patient characteristics at each admission. Groupings based on age at admission were notable for decreasing frequency of malnutrition after 3 years of age, relatively stable frequency of pneumonia across age groups, and increasing frequency of oncologic admissions with increasing age (Fig. 1). Analysis based on the time interval between admission and enrollment in HIV care showed higher frequency of malnutrition early on, relatively stable frequency of pneumonia, and increasing frequency of malaria admissions with increased time in care (Fig. 2). Looking to the impact of immune status on admission diagnoses, malnutrition increased in frequency as a primary diagnosis with increasing immune suppression while the frequency of pneumonia showed less variation across immune categories (Fig. 3). The trends seen relative to the interval between ART initiation and hospitalization were similar to those seen for clinical enrollment with higher frequencies of malnutrition early on, relatively stable frequency of pneumonia, and increasing frequency of malaria admissions with increased time on ART (Fig. 4). 


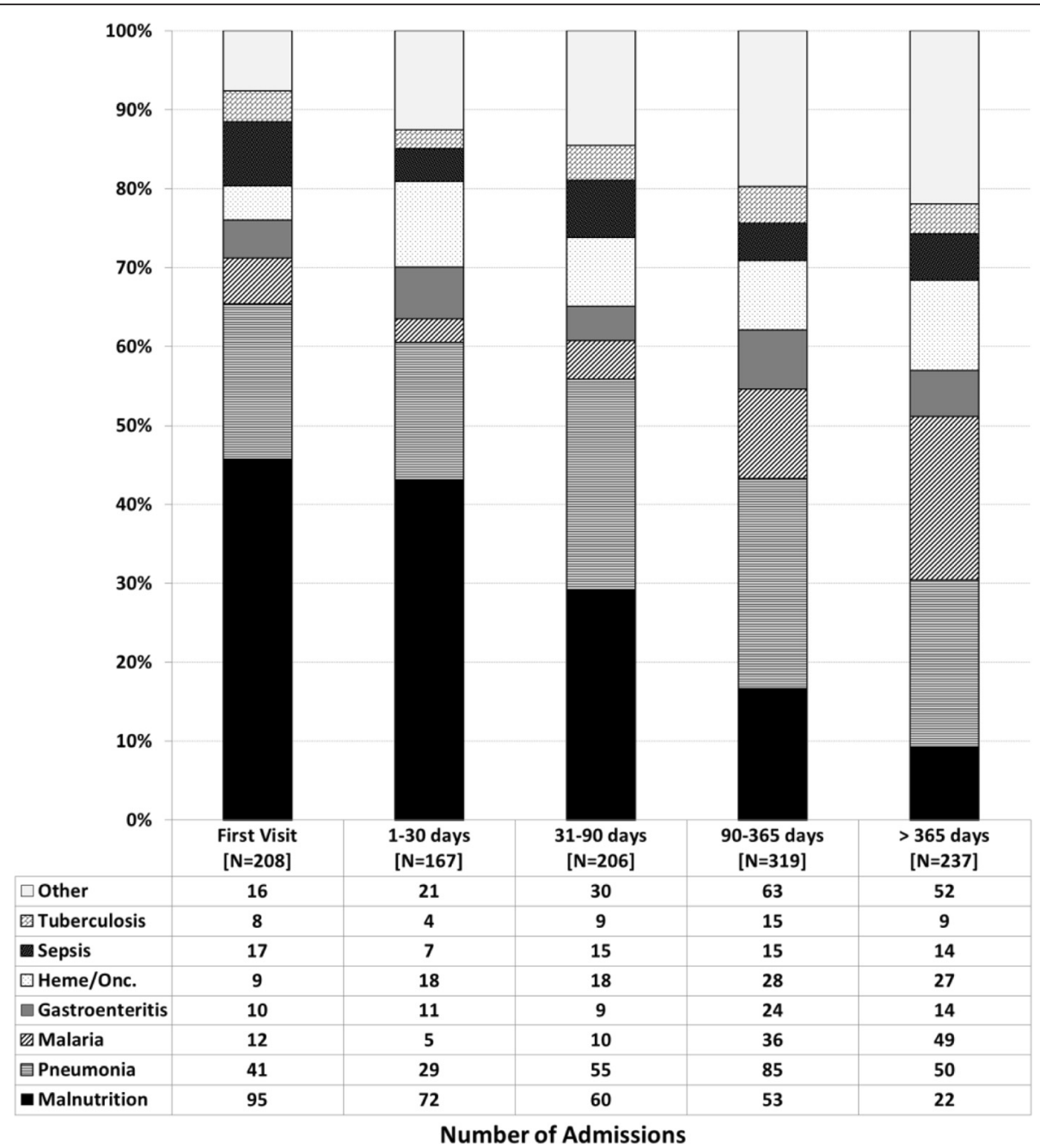

Fig. 2 Stacked bar graph of primary admission diagnosis by time from clinic enrollment, Malawi 2004-2010

\section{Discussion}

This study examines the inpatient admissions from a large cohort of HIV-infected and exposed children enrolled in outpatient HIV care. One of the most striking findings was the high mortality rates (30.8 \%) noted in children who had been admitted. Previous research from the same clinic reported an overall mortality of $4.8 \%$ in pediatric patients on ART [2]. While the cohorts in the studies were not the same, it is clear that patients requiring admission have high relative risk of mortality, highlighting the importance of both in-hospital and post-discharge care.

The clinic lost-to-follow up rate of $10.8 \%$ for this cohort is similar to rates reported in pediatric ART populations from the region $(8.4-11.5 \%)$ [2, 17-19]. Patients admitted from the COE likely benefitted from the close physical proximity of the ward and outpatient site, as well as the shared resources of clinicians, labs, and support staff between the two, allowing for better coordination of inpatient and post-discharge care. In other settings where inpatient and outpatient care are provided in disparate locations by different providers, this linkage may be more problematic and lost-to-follow-up rates after discharge may be higher.

With respect to the demographics and characteristics of clinic patients who were admitted, the high proportion $(49.4 \%)$ of admissions in patients less than two years of age stands out. Previous studies have also shown hospitalizations in HIV-infected children are more common in this age group, and while these trends are similar to pediatric admission trends from this region regardless of HIV, the results stress the need for close monitoring of young children that are enrolled in HIV care [5, 9, 10, 20]. This also supports the need for expanded and improved early infant diagnosis (EID) programs to identify asymptomatic HIV-infected children and enroll them in 


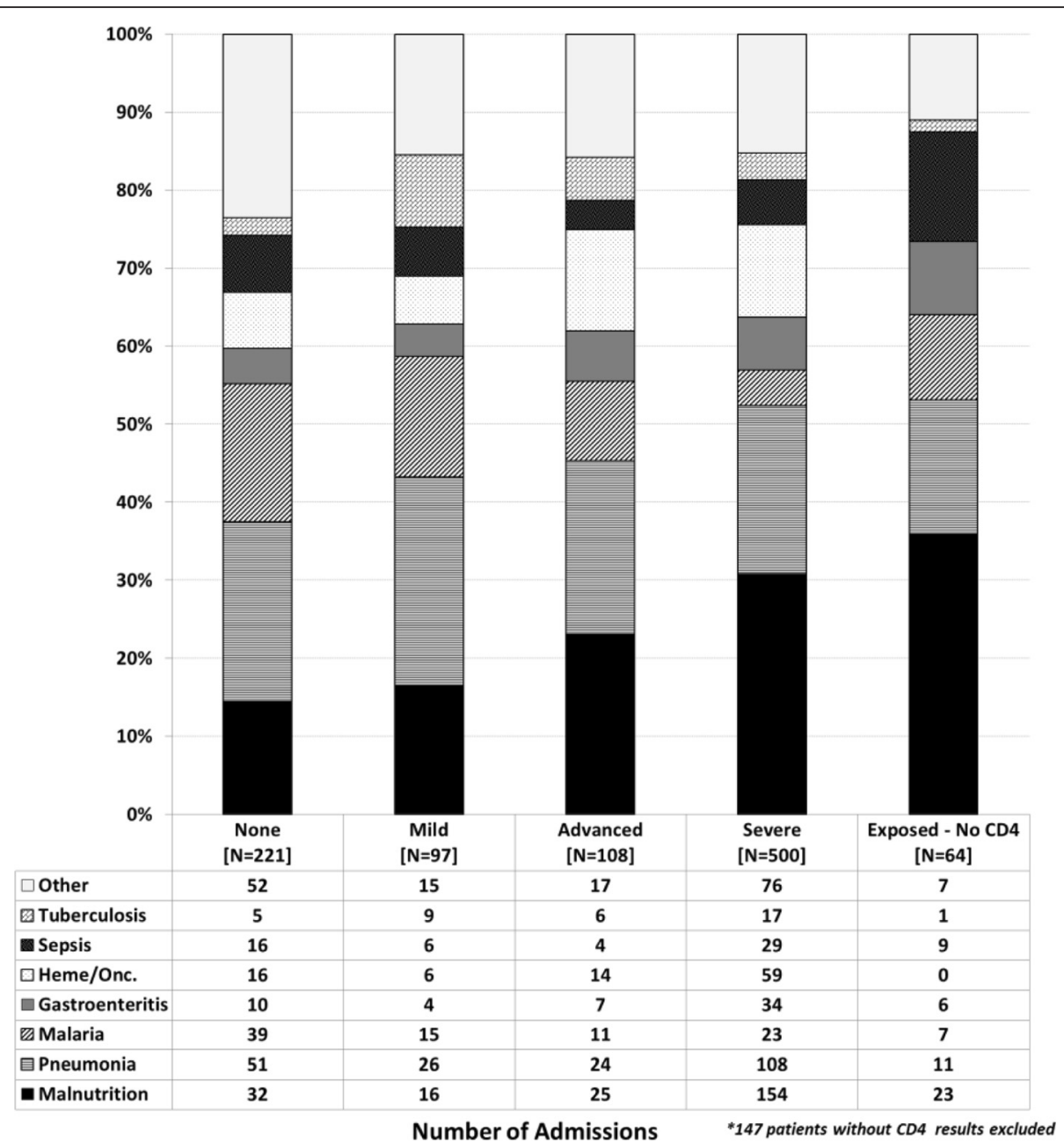

Fig. 3 Stacked bar graph of primary admission diagnosis by immune suppression, Malawi 2004-2010

care prior to disease progression which could require hospitalization.

The large percentage of admissions that occurred in patients who were only recently enrolled in HIV care (18.3\% of admissions occurred on the first day of enrollment into clinic and $33 \%$ within the first month of entry into pediatric HIV care) and in those with evidence of severe immunosuppression (53.5\% had advanced or severe immunologic stage) reflects challenges with late entry into HIV care. These findings also support the need for robust EID programs as well as enhanced provider-initiated testing and counseling programs (PITC) to identify HIV-exposed and infected infants and children and speed their entry into care before they became symptomatic.

A large proportion of admissions occurred in confirmed HIV-infected patients who had not yet started ART, and over half (51.9\%) of these admissions occurred in children who had enrolled in care in the last 30 days. As discussed above, late entry to care certainly contributed to this trend with patients presenting with ART-eligible conditions requiring inpatient care at the time of diagnosis. While our data set did not allow for accurate determination of the proportion of these children who were actually ART-eligible given the timing of CD4 results and evolving national eligibility guidelines over the period of the study, we believe delays in initiation for ART-eligible children already in care also likely contributed to some of these admissions. Prior studies have demonstrated decreased pediatric hospital admission rates in patients who are started on ART $[12,21]$ and these results stress the importance of HIV clinic strategies that accelerate ART eligibility determinations, such as presumptive infant diagnosis, to avoid delays in definitive diagnosis with EID and timely access to CD4 testing. Recent guideline changes granting universal 


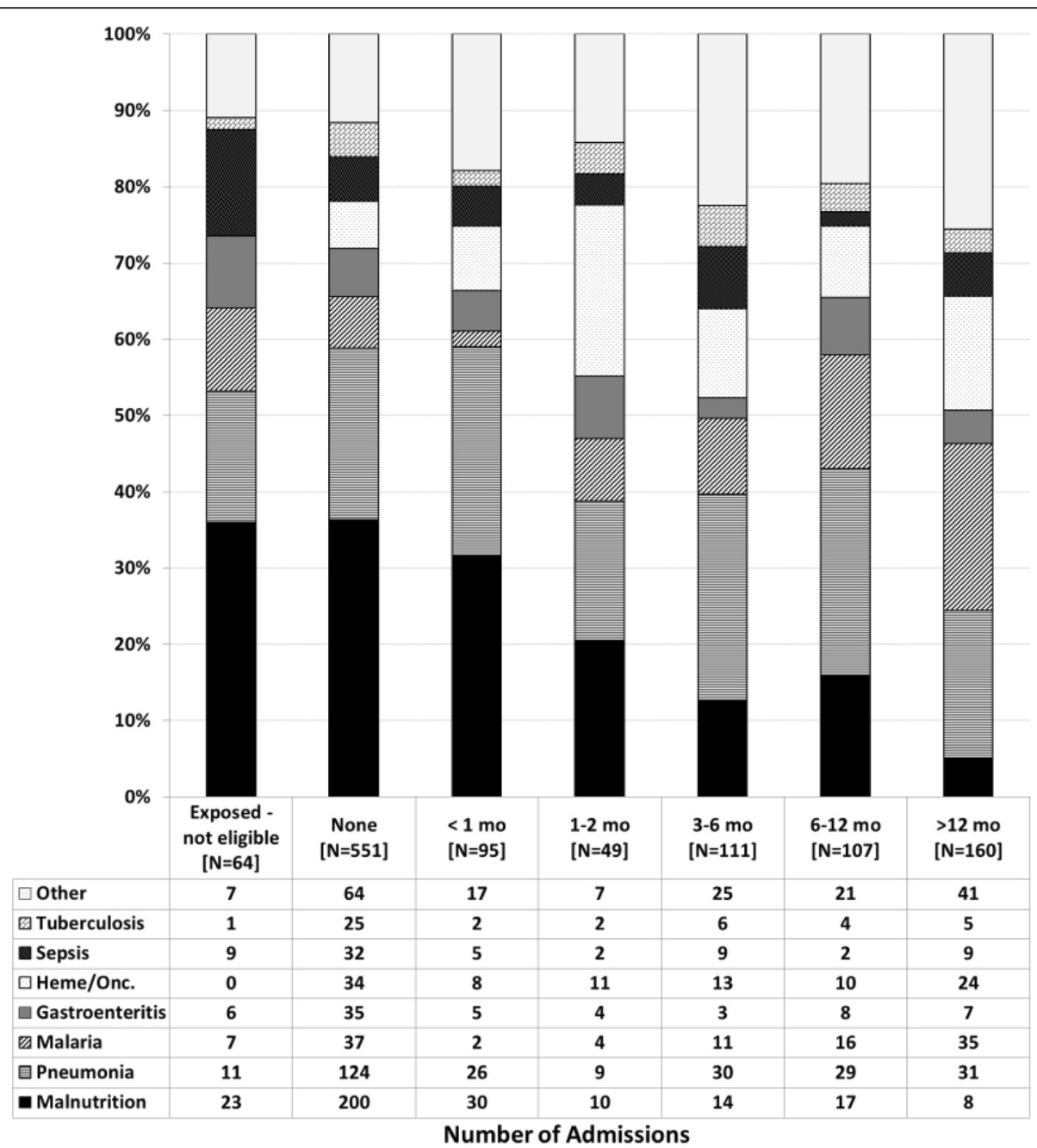

Fig. 4 Stacked bar graph of primary admission diagnosis by time on ART, Malawi 2004-2010

ART access to children under 5 years of age and all HIV-infected patients with TB should also help reduce the time needed to determine ART eligibility. Once eligibility is confirmed, clinic flows that prioritize and expedite pre-ART counseling and education for caregivers are critical.

The most common primary admission diagnosis was acute malnutrition $(26.6 \%$.) It was the most common primary diagnosis in all children less than 3 years with a peak in the 12-23 month age group where it made up $45.0 \%$ of admissions. It also made up the highest proportion of admissions that occurred within the first 3 months from enrollment into HIV care, with a decreasing proportion of admissions with increased time in care. Similarly, acute malnutrition also became a less likely cause of admission the longer a patient had been on ART. Finally, acute malnutrition was the most common cause of admission in exposed infants as well as in HIV-infected children with unknown, severe, and advanced immunologic stage, with a steadily increasing proportion of admissions with increasing immune suppression. These findings demonstrate the importance of routine nutritional screening and treatment as part of comprehensive HIV care and also reinforce the need for routine opt-out PITC, including criteria for presumptive HIV diagnosis, for children diagnosed with acute malnutrition in the sub-Saharan African region.

Pneumonia was the second most common overall primary admission, with the largest observed burden in patients less than 1 year of age. Presumed pneumocystis jiroveci pneumonia is made on clinical grounds in Malawi and was included in the all-cause pneumonia category, possibly explaining the relatively higher rates seen in younger patients, as they are known to be at 
higher risk for this condition [22]. Pneumonia represented a relatively stable proportion of primary admission diagnoses after the first year of life. The trends noted in acute malnutrition related to a time from enrollment in HIV care, immunosuppression, and time on ART were not observed with pneumonia. These results are consistent with data demonstrating the huge burden of pneumonia in all children in this setting, and support the argument that expanded access to pneumococcal (introduced in Malawi after the study period in 2011) and Haemophilus influenza type b (introduced in Malawi before the study period in 2002) immunization is urgently needed, particularly in at-risk HIV-infected children where the effectiveness of these vaccines in preventing invasive disease has been demonstrated [23-25].

Malaria was the third most common primary admission diagnosis, with the highest proportion of admissions noted in the 36-59 month age group (17.2 \%). We hypothesize that in the period after protective maternal antibodies wane around 12 months, malaria is likely a steady cause of illness in this patient population and the observed frequencies in age groups younger than 5 years vary primarily because of the relative burden of other conditions, particularly acute malnutrition. The decreased relative frequency seen in the older groups could be related to acquired immunity from prior infections. The proportion of admissions due to malaria was noted to increase with longer time enrolled in care or on ART, and with improved immune status. This highlights the fact that healthy children with well-managed HIV are still vulnerable to endemic malaria in the region and comprehensive HIV care needs to include malaria education for caregivers and distribution of insecticide-treated bed nets.

This study has several strengths, most notably the large cohort of pediatric patients, the robust, standardized data available in the COE electronic medical records, and additional manual chart review to verify and augment information obtained from database queries. The principal limitation of our study methodology was the reliance on clinic charts alone, as it was not possible to retroactively link patients and trace records with the hospital archives. Some provisional diagnoses may have changed after admission, but unless they were documented in follow-up EMR notes, that information was not captured. In addition, the determination of primary admission diagnoses in patients with multiple problems was at times challenging. Finally, admissions to other hospitals or from other urgent care clinics may have been missed if not reported to COE clinicians and entered into the EMR.

The COE is a referral center for both the Northern and Central regions of the country for Kaposi's sarcoma and other pediatric oncologic diseases, as well as for patients who required second line ART and other complicated cases. It also offers outpatient malnutrition and $\mathrm{TB}$ treatment for its HIV-infected and exposed patients, and the relative percentage of admissions due to these conditions might be higher than what would be seen in a standard high-volume national HIV site. Because some patients had multiple admissions, more chronic conditions such as Kaposi's sarcoma may be overrepresented in comparison to more acute illnesses such as malaria, pneumonia, and gastroenteritis which likely only required one hospitalization.

\section{Conclusion}

The high burden of admissions in children less than 24 months and those newly enrolled in care suggests a need for continued improvement of early infant diagnosis and provider-initiated testing programs to link patients to care before they are symptomatic. Similarly, the high proportion of admissions in those not yet started on ART emphasizes the importance of rapid initiation of ART for eligible pediatric patients. These results reinforce the need for comprehensive care for HIVinfected and exposed children with special attention to nutrition. Strengthened programs for malaria prevention and introduction of pneumococcal vaccine are also needed as part of a comprehensive pediatric HIV care package. The demographics of children in HIV care will be changing as national PMTCT programs yield fewer infected infants and eligibility guidelines move towards universal pediatric ART access, which may result in different hospitalization patterns in the future.

\section{Abbreviations \\ ART: antiretroviral therapy; COE: center of excellence; EID: early infant diagnosis (of HIV); EMR: electronic medical record; HIV: human \\ immunodeficiency virus; IQR: interquartile range; KCH: Kamuzu Central Hospital; MUAC: mid-upper arm circumference; PITC: provider-initiated testing and counseling (for HIV); PMTCT: prevention of mother to child transmission (of HIV); TB: tuberculosis; WHO: World Health Organization.}

\section{Competing interests}

The authors have no financial or non-financial competing interests to report.

\section{Authors' contributions}

CAN contributed to study design, data analysis, and had primary responsibility for manuscript drafting; WCB contributed to study design, data collection, data analysis, and had secondary responsibility for manuscript drafting; ACC had primary responsibility for data analysis and contributed to manuscript drafting and revision; AF contributed to data collection and manuscript revision; YN contributed to manuscript drafting and revision; MB contributed to data collection and manuscript revision; PNK contributed to study design, manuscript revision and gave final approval for submission. All authors read and approved the final manuscript.

\section{Acknowledgments}

The authors thank the clinical teams at the Baylor College of Medicine/ Abbott Fund Children's Clinical Centre of Excellence - Malawi and the Pediatric Ward at Kamuzu Central Hospital who cared for the patients included in this study; Baylor College of Medicine International Pediatric AIDS Initiative senior leadership including Michael Mizwa, Gordon Schutze, Nancy Calles, and Mark Kline; and colleagues at the HIV Department of the Malawi Ministry of Health. 


\section{Author details}

'Baylor Children's Foundation Malawi, Lilongwe, Malawi. ${ }^{2}$ Department of Pediatrics, University of California San Francisco, San Francisco, California, USA. ${ }^{3}$ Department of Pediatrics, University of California Los Angeles, Maputo, Mozambique. ${ }^{4}$ Department of Pediatrics, Baylor College of Medicine, Houston, Texas, USA. ${ }^{5}$ Department of Pediatrics, University of Colorado, Aurora, Colorado, USA.

Received: 4 July 2015 Accepted: 22 January 2016

Published online: 30 January 2016

\section{References}

1. Joint United Nations Programme on HIV/AIDS. Global Report: UNAIDS report on the global AIDS epidemic 2013. Geneva, Switzerland: UNAIDS; 2013.

2. Kabue MM, Buck WC, Wanless SR, Cox CM, McCollum ED, Caviness AC, et al. Mortality and clinical outcomes in HIV-infected children on antiretroviral therapy in Malawi, Lesotho, and Swaziland. Pediatrics. 2012;130:e591-9.

3. Phelps BR, Ahmed S, Amzel A, Diallo MO, Jacobs T, Kellerman SE, et al. Linkage, initiation and retention of children in the antiretroviral therapy cascade: an overview. AIDS. 2013;27 Suppl 2:S207-13.

4. Bedri A, Lulseged S. Clinical description of children with HIV/AIDS admitted at a referral hospital in Addis Ababa. Ethiop Med J. 2001;39(3):203-11.

5. Meyers TM, Pettifor JM, Gray GE, Crewe-Brown H, Galpin JS. Pediatric admissions with human immunodeficiency virus infection at a Regional Hospital in Soweto, South Africa. J Trop Pediatr. 2000:46:224-30.

6. Nathoo KJ, Bannerman CH, Pirie DJ. Patterns of admissions to the paediatric medical wards (1995 to 1996) at Harare Hospital, Zimbabwe. Cent Afr J Med. 1999:45(10):258-63.

7. Rabie H, de Boer A, van den Bos S, Cotton MF, Kling S, Goussard P, et al. Children with human immunodeficiency virus infection admitted to a pediatric intensive care unit in South Africa. J Trop Pediatr. 2007;4:270-3.

8. Chopra M, Stirling S, Wilkinson D, Connolly C, McCoy D. Paediatric admissions to a rural South African hospital: value of hospital data in helping define intervention priorities and allocate district resources. S Afr Med J. 1998;88(6 Suppl):758-8.

9. Rogerson RR, Gladstone M, Callaghan M, Erhart L, Rogerson SJ, Borgstein E, et al. HIV infection among paediatric in-patients in Blantyre, Malawi. Trans R Soc Trop Med Hyg. 2004;98:544-52.

10. Meyers T, Dramowski A, Schneider H, Gardiner N, Kuhn L, Moore D, et al. Changes in paediatric HIV-related hospital admissions and mortality in Soweto, South Africa 1996-2011: light at the end of the tunnel? J Acquir Immune Defic Syndr. 2012;60(5):503-10.

11. McCollum ED, Preidis GA, Kabue MM, Singogo EB, Mwansambo C, Kazembe PN, et al. Task shifting routine inpatient pediatric HIV testing improves program outcomes in urban Malawi: A retrospective observational study. PLoS One. 2010;5(3):e9626.

12. Sudjaritruk T, Oberdorfer P, Puthanakit T, Sirisanthana T, Sirisanthana V. Causes of first hospitalization among 1121 HIV-infected children: comparison of the pre-Pneumocystic jiroveci pneumonia prophylaxis, pre-antirretroviral therapy and antiretroviral therapy periods. Int J STD AIDS. 2012:23:335-9.

13. Malawi Ministry of Health. Malawi antiretroviral treatment programme quarterly report. Results up to 31st December 2010. Lilongwe, Malawi: Malawi MoH; 2010.

14. Preidis GA, McCollum ED, Mwansambo C, Kazembe PN, Schutze GE, Kline MW. Pneumonia and malnutrition are highly predictive of death among African children hospitalized with human immunodeficiency virus infection or exposure early in the era of antiretroviral therapy. J Pediatr. 2011;159(3):484-9.

15. Kim MH, Cox C, Dave A, Draper HR, Kabue M, Schutze GE, et al. Prompt initiation of ART with therapeutic food is associated with improved outcomes in HIV-infected Malawian children with malnutrition. J Acquir Immune Defic Syndr. 2012:59:173-6.

16. World Health Organization Regional Offi ce for South-East Asia, UNICEF. Management of HIV infection and antiretroviral therapy in infants and children: a clinical manual. WHO Technical Publication No. 51. New Delhi, India: WHO SEARO; 2006

17. Fenner L, Brinkhof MW, Keiser O, Weigel R, Cornell M, Moultrie H, et al. Early mortality and loss to follow-up in HIV-infected children starting antiretroviral therapy in Southern Africa. J Acquir Immune Defic Syndr. 2010;54(5):524-32.
18. Bong CN, Yu JK, Chiang HC, Huang WL, Hsieh TC, Schouten EJ, et al. Risk factors for early mortality in children on adult fixed dose combination antiretroviral treatment in a central hospital in Malawi. AIDS. 2007:21(13):1805-10.

19. Ellis J, Molyneux EM. Experience of antiretroviral treatment for HIV-infected children in Malawi: the 1st 12 months. Ann Trop Paediatr. 2007;27(4):261-7.

20. Gordon DM, Frenning S, Draper HR, Kokeb, M. Prevalence and burden of diseases presenting to a general pediatrics ward in Gondar, Ethiopia. J Trop Pediatr. 2013;59(5):350-7.

21. Viani RM, Araneta MRG, Deville JG, Spector SA. Decrease in hospitalization and mortality rates among children with perinatally acquired HIV Type 1 infection receiving highly active antiretroviral therapy. Clin Infect Dis. 2004:39(5):725-31.

22. Mofenson LM, Brady MT, Danner SP, Dominguez KL, Hazra R, Handelsman E, et al. Guidelines for the prevention and treatment of opportunistic infections among HIV-exposed and HIV-infected children: recommendations from CDC, the National Institutes for Health, the HIV Medicine Association of the Infectious Diseases Society of America, the Pediatric Infectious Diseases Society, and the American Academy of Pediatrics. MMWR Recomm Rep. 2009:58(RR-11):1-166.

23. von Gottberg A, de Gouveia L, Madhi SA, du Plessis M, Quan V, Soma K, et al. Impact of conjugate Haemophilus influenzae type $b$ (Hib) vaccine introduction in South Africa. Bull World Health Organ. 2006;84(10):811-8.

24. Klugman KP, Madhi SA, Huebner RE, Kohberger R, Mbelle N, Pierce N. A trial of a 9-valent pneumococcal conjugate vaccine in children with and those without HIV infection. N Engl J Med. 2003:349(14):1341-8.

25. Madhi SA, Kuwanda L, Cutland C, Klugman KP. The impact of a 9-valent pneumococcal conjugate vaccine on the public health burden of pneumonia in HIV-infected and - uninfected children. Clin Infect Dis. 2005;40(10):1511-8

\section{Submit your next manuscript to BioMed Central and we will help you at every step:}

- We accept pre-submission inquiries

- Our selector tool helps you to find the most relevant journal

- We provide round the clock customer support

- Convenient online submission

- Thorough peer review

- Inclusion in PubMed and all major indexing services

- Maximum visibility for your research

Submit your manuscript at www.biomedcentral.com/submit 